\title{
WEAKLY CONTINUOUS FUNCTIONS ON BANACH SPACES NOT CONTAINING $l_{1}$
}

\author{
JOAQUÍN M. GUTIÉRREZ
}

(Communicated by Palle E. T. Jorgensen)

\begin{abstract}
Banach spaces not containing $l_{1}$ are characterized in terms of continuous and holomorphic functions and polynomials which are weakly sequentially continuous and weakly continuous on bounded subsets. An application to (bounded linear) operators is also given.
\end{abstract}

Throughout, $E$ and $F$ are Banach spaces. We write $E^{*}$ for the dual of $E$ and $B_{E}$ for its closed unit ball. $\mathbb{R}, \mathbb{C}$, and $\mathbb{N}$ denote the real, complex, and natural numbers, respectively. By $C_{\mathrm{wk}}(E, F)$ we denote the space of all maps taking weakly convergent sequences in $E$ to convergent ones in $F$, and by $C_{\mathrm{wb}}(E, F)$ we denote the space of those maps whose restrictions to bounded subsets of $E$ are weakly continuous. Clearly, $C_{\mathrm{wb}}(E, F) \subseteq C_{\mathrm{wk}}(E, F)$. When $E$ and $F$ are complex Banach spaces, $H(E, F)$ stands for the space of all holomorphic maps from $E$ to $F$.

For each $k \in \mathbb{N}, \mathscr{P}\left({ }^{k} E, F\right)$ is the space of $k$-homogeneous continuous polynomials from $E$ to $F$. We identify $\mathscr{P}\left({ }^{0} E, F\right)=F$. The space of continuous symmetric $k$-linear mappings from $E \times \stackrel{(k)}{\cdots} \times E$ to $F$ is denoted by $L_{\mathrm{s}}\left({ }^{k} E, F\right)$. The operator $L_{\mathrm{s}}\left({ }^{k} E, F\right) \rightarrow \mathscr{P}\left({ }^{k} E, F\right)$ taking the $k$-linear map $A$ to the polynomial $P$ defined by $P(x)=A(x, \ldots, x)$ is an isomorphism of Banach spaces [14]. If $\mathscr{F}(E, F)$ is a family of continuous maps from $E$ to $F$, then we write

$$
\mathscr{F}_{\alpha}(E, F)=\mathscr{F}(E, F) \cap C_{\alpha}(E, F)
$$

for $\alpha=w b$ or wk. Throughout, if the range space is omitted, it is understood to be the scalar field $\mathbb{K}(=\mathbb{C}$ or $\mathbb{R})$; thus, $\mathscr{P}\left({ }^{k} E\right)=\mathscr{P}\left({ }^{k} E, \mathbb{K}\right)$.

In recent years, many authors have studied such function spaces (see, e.g., [1, $3-6,9,11,13])$. The aim of this note is to give refinements of results from $[5,9]$ characterizing Banach spaces not containing $l_{1}$ in terms of the aforementioned spaces.

We say that a (linear bounded) operator $T: E \rightarrow F$ is completely continuous if it takes weakly convergent sequences in $E$ to convergent ones in $F$. Clearly,

Received by the editors January 21, 1992.

1991 Mathematics Subject Classification. Primary 46E10; Secondary 46G20.

Key words and phrases. Space not containing $l_{1}$, polynomial, holomorphic function, weakly sequentially continuous function.

The author was supported in part by DGKYT Project PB90-0044 (Spain). 
$\mathscr{P}_{\text {wk }}\left({ }^{1} E, F\right)$ is the space of completely continuous operators from $E$ to $F$. On the other hand, $\mathscr{P}_{\text {wb }}\left({ }^{1} E, F\right)$ coincides with the space of compact operators from $E$ to $F$ [6, Proposition 2.5]. We say that $A \subset E$ is a Rosenthal (or conditionally weakly compact) subset if each sequence in $A$ admits of a weak Cauchy subsequence.

The following result, extending a theorem by Rosenthal, is proved in [10].

1. Theorem. Every Rosenthal subset of a Banach space is weakly sequentially dense in its weak closure.

2. Corollary. The space $C_{\mathrm{wk}}(E, F)$ consists of those functions $f: E \rightarrow F$ whose restrictions to Rosenthal subsets of $E$ are weakly continuous.

Proof. If $f \in C_{\mathrm{wk}}(E, F)$ and $A \subset E$ is a Rosenthal subset, then, by Theorem 1 , for every $x \in \bar{A}^{\sigma\left(E, E^{*}\right)}$, there is a sequence $\left(x_{n}\right) \subset A$ converging weakly to $x$. Hence, $\left(f\left(x_{n}\right)\right)$ converges to $f(x)$; so, $f\left(\bar{A}^{\sigma\left(E, E^{*}\right)}\right) \subset \overline{f(A)}$, and $\left.f\right|_{A}$ is weakly continuous.

3. Theorem. Let $E$ be a complex Banach space. The following assertions are equivalent.

(a) E contains no copy of $l_{1}$.

(b) For every $F, C_{\mathrm{wk}}(E, F)=C_{\mathrm{wb}}(E, F)$.

(c) For every complex $F, H_{\mathrm{wk}}(E, F)=H_{\mathrm{wb}}(E, F)$.

(d) For some complex $F, H_{\mathrm{wk}}(E, F)=H_{\mathrm{wb}}(E, F)$.

(e) $H_{\mathrm{wk}}(E)=H_{\mathrm{wb}}(E)$.

Proof. (a) $\Rightarrow$ (b) Since, in a space not containing $l_{1}$, bounded subsets are Rosenthal, it is enough to apply Corollary 2.

(b) $\Rightarrow($ c) $\Rightarrow$ (d) are obvious.

(d) $\Rightarrow$ (e) Suppose there is a function $f \in H_{\mathrm{wk}}(E) \backslash H_{\mathrm{wb}}(E)$. Choose $y \in F$ with $\|y\|=1$, and let $j: \mathbb{R} \rightarrow F$ be given by $j(\lambda)=\lambda y$. Then $j \circ f \in$ $H_{\text {wk }}(E, F) \backslash H_{\text {wb }}(E, F)$.

(e) $\Rightarrow$ (a) Suppose there is a closed subspace $M \subseteq E$ and an isomorphism $S: M \rightarrow l_{1}$, and let $T: l_{1} \rightarrow c_{0}$ be the natural inclusion. By standard arguments, $T$ can be factored through $L_{\infty}[0,1]$ and so extended to an operator $U: E \rightarrow$ $c_{0}$. Since every operator from $L_{\infty}[0,1]$ to $c_{0}$ is weakly compact, and $L_{\infty}[0,1]$ has the Dunford-Pettis property, $U$ is completely continuous. If $\left(e_{n}\right)$ denotes the unit vector basis of $l_{1}$, write $\phi_{n}=2 e_{n} \circ U$. Clearly, the sequence $\left(\phi_{n}\right) \subset E^{*}$ is $\sigma\left(E^{*}, E\right)$-null. Define a function $f: E \rightarrow \mathbb{C}$ by

$$
f(x)=\sum_{n=1}^{\infty}\left(\phi_{n}(x)\right)^{n} \quad(x \in E) .
$$

Then $f$ is well defined and holomorphic [14, 5.5].

We now prove that $f \notin C_{\mathrm{wb}}(E)$. Indeed, otherwise we would have that $g:=f \circ S^{-1} \in C_{\mathrm{wb}}\left(l_{1}\right)$. If $q_{n}$ denotes the $n$th coordinate mapping on $l_{1}$, then

$$
\phi_{n} \circ S^{-1}(y)=2 e_{n} \circ U \circ S^{-1}(y)=2 e_{n} \circ T(y)=2 q_{n}(y) \quad\left(y \in l_{1}\right) \text {; }
$$

so, for $y \in l_{1}$,

$$
g(y)=f \circ S^{-1}(y)=\sum_{n=1}^{\infty}\left(\phi_{n} \circ S^{-1}(y)\right)^{n}=\sum_{n=1}^{\infty}\left(2 q_{n}(y)\right)^{n} .
$$


We now show that $g$ fails to be weakly continuous on the 2-ball. This follows an idea of Professor R. M. Aron which simplifies the author's original proof. Take $\left\{\xi^{1}, \ldots, \xi^{k}\right\} \subset l_{\infty}$, with $\xi^{j}=\left(\xi_{n}^{j}\right)_{n=1}^{\infty}(1 \leq j \leq k)$. We can find an increasing sequence $\left(n_{i}\right) \subset \mathbb{N}$ such that, for each $j(1 \leq j \leq k)$, the sequence $\left(\xi_{n_{i}}^{j}\right)_{i}$ is convergent; therefore, there is an integer $r$ such that, for $p>q \geq r$,

$$
\left|\xi_{n_{p}}^{j}-\xi_{n_{q}}^{j}\right| \leq 1 \quad(1 \leq j \leq k) .
$$

For $y=e_{n_{p}}-e_{n_{r}} \in l_{1}$, with $p>r$, we have

$$
\begin{aligned}
\|y\| & =2, \\
\left|\xi^{j}(y)\right|=\left|\xi_{n_{p}}^{j}-\xi_{n_{r}}^{j}\right| & \leq 1 \quad(1 \leq j \leq k),
\end{aligned}
$$

and

$$
g(y)=2^{n_{p}}+2^{n_{r}}(-1)^{n_{r}} \geq 2 .
$$

Finally, we show that $f \in C_{\mathrm{wk}}(E)$. Indeed, let $L$ be a weakly compact subset of $E$. Then $U(L)$ is compact in $c_{0}$. Set $\varepsilon>0$ and $k \in \mathbb{N}$ with $2^{-k}<\varepsilon$. Since the sequence $\left(e_{n}\right)$ is weak-star null, it converges to 0 uniformly on compact subsets of $c_{0}$; hence, there exists $m \in \mathbb{N}(m \geq k)$ such that

$$
\left|e_{n} \circ U(x)\right|<\frac{1}{4} \quad(x \in L ; n \geq m)
$$

and so

$$
\left|\phi_{n}(x)\right|^{n}<2^{-n} \quad(x \in L ; n \geq m) .
$$

Let $f_{N}(x)=\sum_{n=1}^{N}\left(\phi_{n}(x)\right)^{n}$ for each $N \in \mathbb{N}$; it is clear that $f_{N} \in C_{\mathrm{wb}}(E)$. If $N>M>m$, we have, for every $x \in L$,

$$
\left|f_{N}(x)-f_{M}(x)\right| \leq \sum_{n=M+1}^{N}\left|\phi_{n}(x)\right|^{n}<\sum_{n=M+1}^{\infty} 2^{-n}=2^{-M}<2^{-k}<\varepsilon .
$$

Therefore $\left(f_{N}\right)_{N=1}^{\infty} \subset C_{\mathrm{wb}}(E)$ is a Cauchy sequence in the topology of uniform convergence on weakly compact subsets of $E$. Now $C_{\mathrm{wk}}(E)$ is the completion of $C_{\mathrm{wb}}(E)$ in this topology [9, Proposition 2], so $\left(f_{N}\right)_{N=1}^{\infty}$ converges to $f$, and we conclude that $f \in C_{\mathrm{wk}}(E)$.

The equivalence $(a) \Leftrightarrow(b)$ was proved in [9].

4. Theorem. The following assertions are equivalent.

(a) $E$ contains no copy of $l_{1}$.

(b) For every $F$ and $k \in \mathbb{N}, \mathscr{P}_{\mathrm{wk}}\left({ }^{k} E, F\right)=\mathscr{P}_{\mathrm{wb}}\left({ }^{k} E, F\right)$.

(c) There exists $F$ such that, for every $k \in \mathbb{N}, \mathscr{P}_{\mathrm{wk}}\left({ }^{k} E, F\right)=\mathscr{P}_{\mathrm{wb}}\left({ }^{k} E, F\right)$.

(d) For some $F$ and some $k \in \mathbb{N}(k \geq 2), \mathscr{P}_{\mathrm{wk}}\left({ }^{k} E, F\right)=\mathscr{P}_{\mathrm{wb}}\left({ }^{k} E, F\right)$.

(e) For some $k \in \mathbb{N}(k \geq 2), \mathscr{P}_{\mathrm{wk}}\left({ }^{k} E\right)=\mathscr{P}_{\mathrm{wb}}\left({ }^{k} E\right)$.

Proof. (a) $\Rightarrow$ (b) by Theorem $3(\mathrm{a}) \Rightarrow(\mathrm{b})$.

(b) $\Rightarrow$ (c) $\Rightarrow$ (d) are obvious.

(d) $\Rightarrow$ (e) as in Theorem 3 .

(e) $\Rightarrow$ (a) Suppose $M \subseteq E$ is a closed subspace and $S: M \rightarrow l_{1}$ an isomorphism. Let $T: l_{1} \rightarrow l_{2}$ be the natural inclusion. Since $T$ is absolutely summing [12, Theorem 2.b.6], we can apply the Grothendieck-Pietsch domination theorem $[8$, p. 60$]$. Then there exists a regular Borel probability measure $\mu$ defined on a compact space such that $T$ factors through $L^{\infty}(\mu)$. By the 
injectivity of $L^{\infty}(\mu), T$ extends to an operator $V: E \rightarrow l_{2}$ which is completely continuous by the Dunford-Pettis property of $L^{\infty}(\mu)$. For $x \in E$, write $V(x)=\left(V_{n}(x)\right)_{n=1}^{\infty} \in l_{2}$. For each integer $k \geq 2$, define $P_{k}: E \rightarrow \mathbb{K}$ by

$$
P_{k}(x)=\sum_{n=1}^{\infty}\left(V_{n}(x)\right)^{k} .
$$

$P_{k}$ is the product of the maps

$$
E \stackrel{V}{\rightarrow} l_{2} \stackrel{I}{\rightarrow} l_{k} \stackrel{W}{\rightarrow} \mathbb{K}
$$

where $I$ is the natural inclusion and $W$ the $k$-homogeneous polynomial given by

$$
W(\xi)=\sum_{n=1}^{\infty}\left(\xi_{n}\right)^{k} \quad \text { for } \xi=\left(\xi_{n}\right) \in l_{k} .
$$

Hence, $P_{k} \in \mathscr{P}\left({ }^{k} E\right)$. Moreover, since $V$ is completely continuous, $P_{k} \in$ $\mathscr{P}_{\text {wk }}\left({ }^{k} E\right)$.

Let us now see that $P_{k} \notin \mathscr{P}_{\mathrm{wb}}\left({ }^{k} E\right)$; otherwise we would have $R_{k}:=P_{k} \circ S^{-1} \in$ $\mathscr{P}_{\text {wb }}\left({ }^{k} l_{1}\right)$. If $q_{n}$ is the $n$th coordinate map on $l_{1}$, we have

$$
R_{k}(y)=P_{k} \circ S^{-1}(y)=\sum_{n=1}^{\infty}\left(V_{n} \circ S^{-1}(y)\right)^{k}=\sum_{n=1}^{\infty}\left(q_{n}(y)\right)^{k} \quad\left(y \in l_{1}\right) .
$$

The continuous symmetric $k$-linear map $A_{k}$ associated to $R_{k}$ is given by

$$
A_{k}\left(y_{1}, \ldots, y_{k}\right)=\sum_{n=1}^{\infty} q_{n}\left(y_{1}\right) \cdots q_{n}\left(y_{k}\right) \quad\left(y_{1}, \ldots, y_{k} \in l_{1}\right) .
$$

Let $C_{k}: l_{1} \rightarrow L_{\mathrm{s}}\left({ }^{(k-1} l_{1}\right)$ be the operator defined by

$$
C_{k}(x)\left(y_{1}, \ldots, y_{k-1}\right)=A_{k}\left(x, y_{1}, \ldots, y_{k-1}\right) \quad\left(x, y_{1}, \ldots, y_{k-1} \in l_{1}\right) \text {. }
$$

Then, if $\left(e_{n}\right)$ is the unit vector basis in $l_{1}$, we have, for $n \neq m$,

$$
\begin{aligned}
\left\|C_{k}\left(e_{n}\right)-C_{k}\left(e_{m}\right)\right\| & \geq\left|C_{k}\left(e_{n}\right)\left(e_{n},{ }^{(k-1)}, e_{n}\right)-C_{k}\left(e_{m}\right)\left(e_{n}, \stackrel{(k-1)}{\cdots}, e_{n}\right)\right| \\
& =\left|A_{k}\left(e_{n},\left({ }^{k}\right), e_{n}\right)-A_{k}\left(e_{m}, e_{n},{ }^{(k-1)}, e_{n}\right)\right|=1 ;
\end{aligned}
$$

therefore, $C_{k}$ is not compact. Applying [5, Theorem 2.9], we conclude that $R_{k} \notin \mathscr{P}_{\mathrm{wb}}\left({ }^{k} l_{1}\right)$.

The same argument would give another proof of Theorem 3 on holomorphic functions. Nevertheless, we present both since the one of Theorem 3 only needs basic tools from Banach space theory and gives a concrete example of a holomorphic function that could be useful in other applications.

The equivalence $(\mathrm{a}) \Leftrightarrow(\mathrm{b})$ is proved in [5, Proposition 2.12 and following comment]. As far as we know, (c), (d), and (e) are new.

Assertions (d) and (e) of the last theorem show a different behaviour of polynomials and operators. Odell proved (see [15, p. 377]) that $E$ contains no copy of $l_{1}$ if and only if every completely continuous operator on $E$ is compact. Theorem 4, however, is no longer true for $k=1$ in (e), since for every $E, \mathscr{P}_{\mathrm{wk}}\left({ }^{1} E\right)=\mathscr{P}_{\mathrm{wb}}\left({ }^{1} E\right)=E^{*}$. Even if $F$ were restricted in (d) to be infinite dimensional, the theorem would fail for $k=1$. Indeed, it is known [7, 
Proposition 3.7] that $E$ contains no complemented copy of $l_{1}$ if and only if there exists an infinite-dimensional $F$ such that every completely continuous operator from $E$ to $F$ is compact.

Finally, we give a corollary on operators. We say that an operator $T: E \rightarrow E^{*}$ is symmetric if it verifies

$$
\langle y, T x\rangle=\langle x, T y\rangle \quad(x, y \in E) .
$$

The symmetric operators are studied in $[2, \S 8]$ in relation to spectral properties of algebras of analytic functions and Arens regularity. It is an open problem whether the fact that every symmetric operator from $E$ to $E^{*}$ is weakly compact implies that every operator from $E$ to $E^{*}$ is weakly compact too. Here we give an answer to a similar question.

5. Corollary. The following assertions are equivalent.

(a) E contains no copy of $l_{1}$.

(b) Every completely continuous operator on $E$ is compact.

(c) Every completely continuous operator from $E$ to $E^{*}$ is compact.

(d) Every symmetric completely continuous operator from $E$ to $E^{*}$ is compact.

Proof. (a) $\Rightarrow(b)$ is included in $(a) \Rightarrow(b)$ of Theorem 4.

(b) $\Rightarrow$ (c) $\Rightarrow$ (d) are obvious.

(d) $\Rightarrow$ (a) Suppose $E$ contains an isomorphic copy of $l_{1}$. As in the proof of Theorem 4, we can find a completely continuous operator $V: E \rightarrow l_{2}$ extending the inclusion $T: l_{1} \rightarrow l_{2}$. For every $x \in E$ we write $V(x)=\left(V_{n}(x)\right)_{n=1}^{\infty} \in l_{2}$ and define the 2-homogeneous polynomial $P: E \rightarrow \mathbb{K}$ by

$$
P(x)=\sum_{n=1}^{\infty}\left(V_{n}(x)\right)^{2} .
$$

Let $C: E \rightarrow E^{*}$ be the associated operator given by

$$
\langle y, C(x)\rangle=\sum_{n=1}^{\infty} V_{n}(x) V_{n}(y) \quad(x, y \in E) .
$$

Since $P \notin \mathscr{P}_{\mathrm{wb}}\left({ }^{2} E\right), C$ is not compact [5, Theorem 2.9]. Obviously, $C$ is symmetric, and if $\left(x_{m}\right)_{m=1}^{\infty} \subset E$ is a weakly null sequence, then

$$
\begin{aligned}
\left\|C\left(x_{m}\right)\right\| & =\sup \left\{\left|\left\langle y, C\left(x_{m}\right)\right\rangle\right|: y \in B_{E}\right\} \\
& =\sup \left\{\left|\left\langle V\left(x_{m}\right), V(y)\right\rangle\right|: y \in B_{E}\right\} \leq\left\|V\left(x_{m}\right)\right\| \cdot\|V\| .
\end{aligned}
$$

Since $V$ is completely continuous, so is $C$.

The equivalence $(a) \Leftrightarrow(b)$ is Odell's theorem [15, p. 377].

\section{REFERENCES}

1. R. M. Aron, Weakly uniformly continuous and weakly sequentially continuous entire functions, Advances in Holomorphy, Math. Studies, vol. 34, North-Holland, Amsterdam, 1979, pp. 47-66.

2. R. M. Aron, B. J. Cole, and T. W. Gamelin, Spectra of algebras of analytic functions on a Banach space, J. Reine Angew. Math. 415 (1991), 51-93. 
3. R. M. Aron, J. Diestel, and A. K. Rajappa, Weakly continuous functions on Banach spaces containing $l_{1}$, Banach Spaces (Proceedings, Missouri 1984), Lecture Notes in Math., vol. 1166, Springer-Verlag, Berlin, 1985, pp. 1-3.

4. R. M. Aron and C. Hervés, Weakly sequentially continuous analytic functions on a Banach space, Functional Analysis, Holomorphy and Approximation Theory II, Math. Studies, vol. 86, North-Holland, Amsterdam, 1984, pp. 23-38.

5. R. M. Aron, C. Hervés, and M. Valdivia, Weakly continuous mappings on Banach spaces, J. Funct. Anal. 52 (1983), 189-204.

6. R. M. Aron and J. B. Prolla, Polynomial approximation of differentiable functions on Banach spaces, J. Reine Angew. Math. 313 (1980), 195-216.

7. E. M. Bator, P. Lewis, and D. Race, Some connections between Pettis integration and operator theory, Rocky Mountain J. Math. 17 (1987), 683-695.

8. J. Diestel, Sequences and series in Banach spaces, Graduate Texts in Math., vol. 92, SpringerVerlag, Berlin, 1984.

9. J. Ferrera, J. Gómez, and J. G. Llavona, On completion of spaces of weakly continuous functions, Bull. London Math. Soc. 15 (1983), 260-264.

10. M. González and J. M. Gutiérrez, The compact weak topology on a Banach space, Proc. Roy. Soc. Edinburgh Sect. A 120 (1992), 367-379.

11. J. A. Jaramillo and J. G. Llavona, Homomorphisms between algebras of continuous functions, Canad. J. Math. 41 (1989), 132-162.

12. J. Lindenstrauss and L. Tzafriri, Classical Banach spaces, I. Sequence spaces, Ergeb. Math. Grenzgeb. (3), vol. 92, Springer-Verlag, Berlin, 1977.

13. J. G. Llavona, Approximation of continuously differentiable functions, Math. Studies, vol. 130, North-Holland, Amsterdam, 1986.

14. J. Mujica, Complex analysis in Banach spaces, Math. Studies, vol. 120, North-Holland, Amsterdam, 1986.

15. H. P. Rosenthal, Point-wise compact subsets of the first Baire class, Amer. J. Math. 99 (1977), 362-378.

Departamento de Matemática Aplicada, ETS de Ingenieros Industriales, Universidad Politécnica de Madrid, C. José Gutiérez Abascal 2, 28006 Madrid, Spain

E-mail address: c0550001@emdupm11.bitnet 